\title{
SUBSTRATE-MEDIATED INTERACTION ON AG(111) SURFACES FROM FIRST PRINCIPLES
}

\author{
KRISTEN A. FICHTHORN \\ The Pennsylvania State University \\ University Park, PA 16802 USA \\ AND \\ MATTHIAS SCHEFFLER \\ Fritz-Haber-Institut der Max-Planck-Gesellschaft \\ D-14 195 Berlin-Dahlem, Germany
}

\begin{abstract}
When two or more atoms bind to a solid surface, the substrate can mediate an interaction between them. In this paper, we use densityfunctional theory to quantify the substrate-mediated pair interaction between two adatoms on a compressively strained $\operatorname{Ag}(111)$ surface and on unstrained $\mathrm{Ag}(111)$. On the strained surface, the elastic interaction is significant over the short range and leads to a net attraction between two adatoms. However, at the longest distances probed, the interaction is primarily electronic and repulsive. The repulsion can be as strong at $50 \mathrm{meV}$, and it forms a ring-like structure around an atom. On unstrained $\operatorname{Ag}(111)$, the interaction is primarily electronic in origin and it is weak relative to the interaction found on the strained surface. We calculate the energy barrier for an isolated atom to diffuse in each of these systems. For the strained surface, the magnitude of the diffusion barrier is comparable to that of the adsorbate interaction. We discuss the implications of our findings for growth at surfaces.
\end{abstract}

\section{Introduction}

Adsorbate-adsorbate interactions at surfaces play a key role in controlling the structure and properties of interfacial materials. Such interactions can be classified as through-space (direct) or through-substrate (indirect), although a clear distinction is not possible [1]. Quantification of the indirect, 
substrate-mediated interaction between two adsorbates is the primary subject of this paper.

Substrate-mediated interactions can have an elastic and an electronic component. Elastic interactions arise when an adsorbed atom perturbs the positions of surrounding surface atoms [2]. Additionally, an adsorbate may perturb the charge density near the surface and alter the binding energies of neighboring adsorbates - giving rise to an electronic interaction $[1,3,4,5,6]$. The electronic interaction is oscillatory in nature and, in the limit that the separation $d$ between two adsorbates is much greater than the inverse Fermi wavevector $\mathbf{k}_{F}^{-1}$, the interaction energy $\Delta E$ is predicted [5] to have the form

$$
\Delta E \sim \frac{\cos \left(2 \mathbf{k}_{F} d\right)}{d^{x}}
$$

where $x=2$ if the substrate has a surface state and $x=5$ in the absence of a surface state. Oscillatory, charge-density waves associated with the electronic interaction have been imaged in scanning-tunneling microscopy (STM) studies of noble-metal surfaces [7, 8], which posses a surface state. For example, on $\operatorname{Ag}(111), \mathbf{k}_{F}=0.08 \AA$ [8] and charge density waves can be imaged at distances greater than $70 \AA$ [8] in STM studies.

Electronic, substrate-mediated interactions should also be manifested at "intermediate" distances between the short separations required for chemical bonds and the long separations for which Eq. 1 is applicable. For example, Einstein and Schrieffer showed in tight-binding calculations that the intermediate-range interaction is present and is, in fact, more significant than the long-range interaction [3]. Recent, low-temperature STM studies also indicate that this is the case [9] for $\mathrm{Cu}$ adatoms on $\mathrm{Cu}(111)$. The intermediate range is accessible to modern, electronic-structure calculations. In this study, we use density-functional-theory (DFT) calculations to quantify the substrate-mediated interaction between two adatoms on a (compressively) strained and on an unstrained $\operatorname{Ag}(111)$ surface. We discuss the ramifications of these interactions for adsorbate diffusion and growth at surfaces.

\section{Model and Methods}

Total energies and forces are calculated using the DFT code described by Bockstedte et al. [10], employing fully separable, norm-conserving pseudopotentials of the Kleinman-Bylander form [11]. These were generated using the code developed by Fuchs and Scheffler [12]. For our calculations with $\mathrm{Ag}$, the $s$ pseudopotential component is taken as local and we use $p$ and $d$ projectors. The $4 d$-electrons are taken as valence states and their wave functions are expanded in plane waves, which truncate at a cut-off energy 
of $E_{c u t}=50$ Ry for our calculations. We use the generalized gradient approximation (GGA) of Perdew, Burke, and Ernzerhof (PBE) [13] for the exchange-correlation functional.

The supercell approach is used to describe the surface. For most results presented here, the surface is modeled as a repeating $(4 \times 2)$ slab with 16 atoms per unit cell and a thickness of four layers. The vacuum spacing is five interlayer distances, or $\sim 12 \AA$. Adsorbed atoms are placed on one side of the slab and field effects due to the asymmetry are correctly treated in the code by a compensating dipole field. For some of the adsorbate interactions probed, it is expedient to utilize smaller slabs. Thus, we also use four-layer $(2 \times 2),(4 \times 1)$, and $(2 \times 1)$ slabs for some of the calculations. For all of the main results presented here, the $\mathbf{k}$-space integration is performed with the equivalent of 4 special $\mathbf{k}$ points in the full, surface Brillouin zone of the $(4 \times 2)$ slab. To improve $\mathbf{k}$-space integration, the electronic states are occupied according to a Fermi distribution with $k_{B} T=0.1 \mathrm{eV}$ and the total energies are extrapolated to zero temperature.

To verify the accuracy of the pseudo-potential, we calculated the lattice constant of bulk $\mathrm{Ag}$ in its ground state. For these calculations, we use $E_{c u t}=50 \mathrm{Ry}$ and $182 \mathrm{k}$ points in the irreducible portion of the Brillouin zone. We find a lattice constant of $a_{0}=4.20 \AA$, which is within $1 \%$ of the values found by $\mathrm{Yu}$ and Scheffler [14] and by Ratsch et al. [15], who both used DFT with the GGA functional of Perdew et al. [16]. As an additional test of the accuracy, we calculated the interlayer relaxation $d_{12}$ of the first layer of $\mathrm{Ag}(111)$. For these calculations, the first layer atoms are relaxed until the forces on them are less than $0.005 \mathrm{eV} / \AA$. We find that the first layer relaxes inward by $2.0 \%$ relative to the bulk, interlayer spacing $d_{b}$, in good agreement with DFT local-density approximation (LDA) results by Narasimhan and Scheffler [17], who found an inward relaxation of $1.7 \%$, and by Xie, et al. [18], who found an inward relaxation of $1.0 \%$. Mediumenergy ion scattering experiments indicate that $d_{12}$ contracts by $2.5 \%$ relative to $d_{b}$ at temperatures below $670 \mathrm{~K}$ [19]. A more recent low-energy electron diffraction investigation indicates no contraction of $d_{12}$ occurs on this surface [20]. In previous theoretical studies of $\operatorname{Ag}(111)[15,17,18]$, it was found that differences between $d_{23}$ and $d_{b}$ are negligible. Thus, in the calculations presented here, $d_{23}$ is fixed to $d_{b}$.

We study adsorbate interactions on both $\operatorname{Ag}(111)$ and on a strained $\operatorname{Ag}(111)$ surface. For the strained $\operatorname{Ag}(111)$ surface, we employ a bulk lattice constant of $4.01 \AA$. This value is taken from the results of DFT-GGA calculations of the lattice constant of Pt by Ratsch, Seitsonen and Scheffler [15]. We choose this substrate to address experimental issues related to $\mathrm{Ag}$ epitaxy on a $\mathrm{Pt}(111)$ substrate covered by a monolayer (ML) of $\operatorname{Ag}[15,21,22,23]$. To describe the bare, strained $\operatorname{Ag}(111)$ substrate, the 
top layer of atoms is relaxed and the rest are fixed to the strained, bulk positions. This results in an outward relaxation of the top layer by 9.0 $\%$ relative to $d_{b}$ for the strained substrate. The outward relaxation allows for relief of the strain induced by the bulk lattice, which is compressed by $4.61 \%$ relative to bulk $\mathrm{Ag}$, as it is described by our DFT calculations with the GGA.

The total interaction energy $\Delta E$ for a periodic slab containing $N$ adatoms, of which $M$ are at binding site $a$ and $(N-M)$ are at binding site $b$, is given by

$$
\Delta E=E_{S+N}^{a, b}-M E_{S+1}^{a}-(N-M) E_{S+1}^{b}+(N-1) E_{S} .
$$

Here, $E_{S+N}^{a, b}$ is the total energy of a slab with $N$ adatoms, $E_{S+1}^{a}$ and $E_{S+1}^{b}$ are the total energies of slabs containing one adatom, and $E_{S}$ is the total energy of a bare slab. We obtain $E_{S}$ from the calculations described above. In an attempt to isolate the electronic component of $\Delta E$, we obtain $E_{S+N}^{a, b}$, $E_{S+1}^{a}$, and $E_{S+1}^{b}$ as follows: A single atom is placed in a binding site (an fcc or hcp three-fold hollow site) on a relaxed (as described above) bare slab and its height is optimized with respect to the fixed substrate. ¿From this calculation, we obtain $E_{S+1}^{a}$ and $E_{S+1}^{b}$. To calculate $E_{S+N}^{a, b}$, adatoms are placed in binding sites on the relaxed (and fixed) substrate with heights fixed to values from the single-adatom calculations. We use this procedure to obtain most of the results described below. Additionally, to resolve the elastic contribution to $\Delta E$, we obtain $E_{S+N}^{a, b}, E_{S+1}^{a}$, and $E_{S+1}^{b}$ by simultaneously relaxing the adatoms and the first-layer substrate atoms for a few trial configurations.

In the DFT supercell approach, the total interaction energy $\Delta E$ is comprised of interactions between different adatoms in the slab and interactions between adatoms in the slab and the periodic-image adatoms. We can express $\Delta E$ in terms of these interactions using the lattice-gas Hamiltonian approach (see, e.g.[24]), which yields

$$
\Delta E=\frac{1}{2} \sum_{i, j} V^{(2)}\left(d_{i, j}\right) \sigma_{i} \sigma_{j}+\frac{1}{3} \sum_{i, j, k} V^{(3)}\left(d_{i, j}, d_{i, k}, d_{j, k}\right) \sigma_{i} \sigma_{j} \sigma_{k}+\ldots
$$

Here, the summations run over all sites $i$ in the slab and all sites $j$ and $k$ in the supercell (which includes both the slab and its periodic images), $\sigma_{m}$ is unity if site $m(m=i, j, k)$ is occupied and zero, otherwise, $d_{m, n}$ is the distance between sites $m$ and $n, V^{(2)}\left(d_{i, j}\right)$ is the pair interaction between two adatoms on sites $i$ and $j$, and $V^{(3)}\left(d_{i, j}, d_{i, k}, d_{j, k}\right)$ is the trio interaction between three adatoms on sites $i, j$, and $k$. We neglect higher-order interactions. Another assumption implicit in Eq. 3 is that the interaction between adatoms at a fixed distance is independent of whether these atoms 


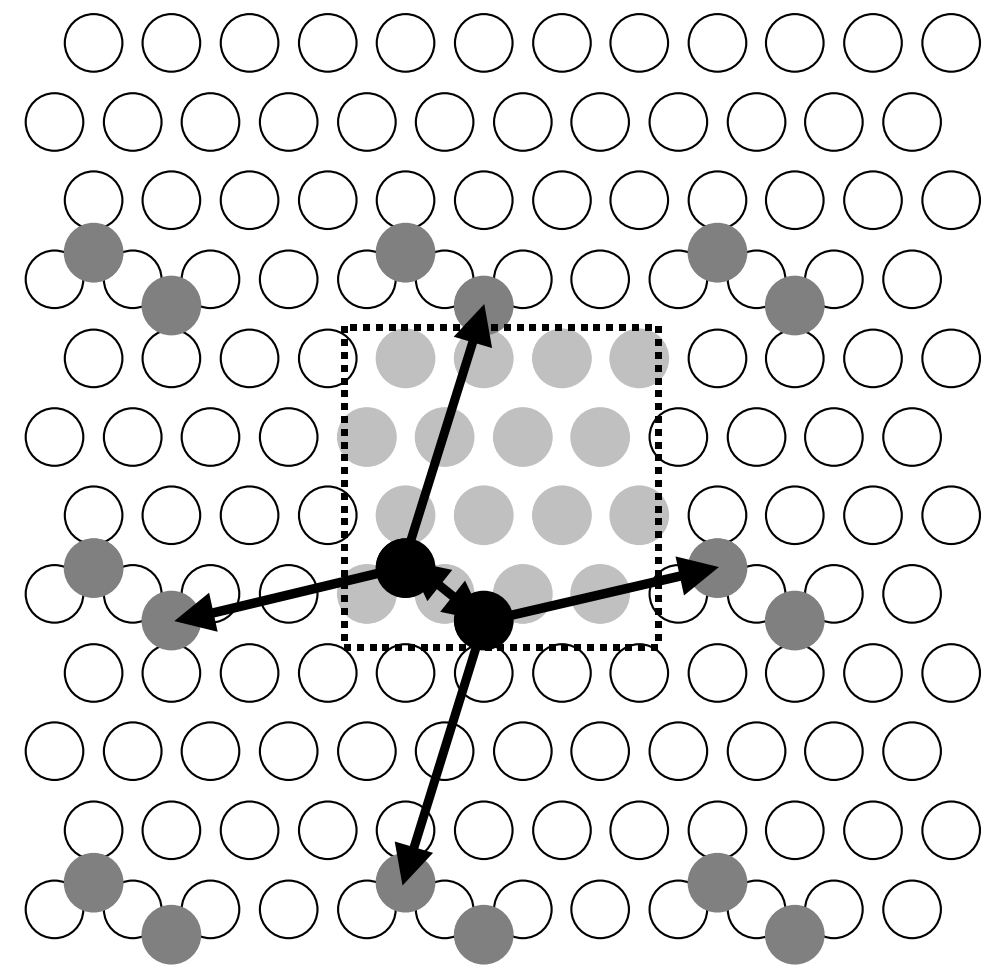

Figure 1. Sample lattice-gas Hamiltonian configuration. Atoms in the slab are enclosed in the dashed box. The lines with arrows indicated relevant distances between adatoms in the slab and periodic-image adatoms.

occupy fcc or hcp sites. Actually, in the results presented below, interactions between two adatoms occupying the same type of binding site are always obtained for adatoms on fcc sites. We performed one test of this assumption for two adatoms on hcp sites. This calculation showed that the interaction was identical to that found for atoms on fcc sites. Finally, in calculations designed to resolve the electronic interaction (which comprise the bulk of the results presented here), the adatom binding energies on fcc and hcp sites are virtually equal: The fcc site is favored by less than $3 \mathrm{meV}$.

To illustrate our approach, we show one of the structures probed in our calculations in Fig. 1 . In this structure, two adatoms are in the $(4 \times 2)$ surface unit cell at a separation of $3.43 \AA$ (distance 2 , as can be inferred from Fig. 4 below). Each of these adatoms has two, neighboring, periodic-image adatoms at a distance of $9.08 \AA$ (distance 12 shown on Fig. 4 below). From Eq. 3, $\Delta E$ for this configuration is comprised of one pair interaction at distance 2 and two at distance 12. Thus, for a given adatom configuration, we express $\Delta E$ as a sum of pair and trio interactions with unknown 
coefficients. From 13 different configurations, we obtain a system of linear equations and solve these for pair-interaction coefficients up to the $13^{\text {th }}$ neighbor. We assume that all other interaction coefficients are zero.

\section{Results and Discussion}

Before proceeding to our results, we discuss our tests of their accuracy. We test the convergence of our results with respect to $E_{c u t}$, the number of $\mathbf{k}$ points, the slab thickness, and the exchange-correlation functional for different adsorbate configurations on $\operatorname{Ag}(111)$ and strained $\operatorname{Ag}(111)$. The adsorbate configurations in these tests are chosen for two major reasons. First, for each configuration, the total interaction energy is composed of interactions at a single, adsorbate-pair distance. Thus, it is possible to extract pair interactions without solution of the full system of equations for the lattice-gas Hamiltonian. A second reason for choosing these configurations is that they posses a single mirror plane - the highest symmetry that could be achieved here. The (relatively) high symmetry makes it possible to retain up to $16 \mathbf{k}$ points in the full surface Brillouin zone of a $(4 \times 2)$ unit cell, so that we can assess the convergence of the $\mathbf{k}$-point summations for these configurations. For configurations of lower symmetry, 4 k-points in the $(4 \times 2)$ unit cell is the maximum that we could use with the computational resources available to us. In Table 1 we show the influence of the cut-off energy, the slab thickness, and the number of $\mathbf{k}$ points on three, pair-interaction coefficients for $\mathrm{Ag}$ adatoms on $\mathrm{Ag}(111)$. The distances for the pair interactions can be seen below in Fig. 4. For each result in Table 1, each of the three energies necessary to obtain the pair-interaction coefficient (cf., Eq. 2) is obtained with the indicated cut-off energy, slab thickness, and number of $\mathbf{k}$ points.

TABLE 1. Convergence tests for $V^{(2)}(d)$.

\begin{tabular}{ccccc}
\hline Distance, $d$ & $N_{\mathbf{k}}$ & $E_{\text {cut }}$, Ry & Layers & $V^{(2)}(d), \mathrm{eV}$ \\
\hline 5 & 4 & 50 & 4 & -0.012 \\
5 & 4 & 60 & 4 & -0.015 \\
5 & 4 & 50 & 5 & -0.005 \\
5 & 16 & 50 & 4 & 0.012 \\
4 & 4 & 50 & 4 & 0.004 \\
4 & 16 & 50 & 4 & 0.001 \\
9 & 4 & 50 & 4 & 0.005 \\
9 & 16 & 50 & 4 & -0.001 \\
\hline
\end{tabular}


From Table 1, we see that the tests for distance 5 indicate satisfactory convergence with respect to the cut-off energy and the slab thickness. The results of our $\mathbf{k}$-point test for distance 5 are less satisfying and there is a (relatively) large difference between the pair-interaction coefficients obtained with 4 and $16 \mathrm{k}$ points. To further probe the convergence of our results with respect to the $\mathbf{k}$-point summation, we investigated two additional adsorbate configurations containing distances 4 and 9. For these configurations, differences in the pair-interaction coefficients are small and indicate a satisfactory $\mathbf{k}$-point convergence. It appears that distance 5 is somehow unique. We will discuss this configuration in more detail below.

To assess the dependence of our results on the exchange-correlation functional, we obtain pair-interaction coefficients for three, different adatom separations on strained $\operatorname{Ag}(111)$ using the LDA [25]. The pseduopotentials for these calculations are generated as described above for the GGA calculations, except that the LDA is used for the exchange-correlation functional. We test this pseudopotential by calculating the bulk lattice constant for $\mathrm{Ag}$ using the same conditions as for the GGA calculations. We find $a_{0}=4.06$ $\AA$, which is in exact agreement with the value found by Narasimhan and Scheffler [17] and within 1\% of the value found by Ratsch, et al. [15]. For strained $\mathrm{Ag}(111)$, we employ a lattice constant with a value of $3.92 \AA$, as found by Ratsch et al. [15] in DFT-LDA calculations of the lattice constant for bulk Pt.

In Fig. 2, we show a comparison of the LDA and GGA results. Here, we see a small difference between the pair-interaction coefficients for distances 4 and 9 and a (relatively) large difference between the LDA and the GGA at distance 5. Some of the discrepancy between the LDA and GGA results can likely be attributed to differences in strain. The lattice in the GGA calculations is compressively strained by $4.61 \%$ relative to the bulk, while the strain for the LDA system is $3.21 \%$ (for a reference, the experimental strain is $4.2 \%$ [22]). As we will discuss in more detail below, we find that pair interactions on $\mathrm{Ag}(111)$ follow the same trend as those on compressively strained $\mathrm{Ag}(111)$, but they are consistently lower. A comparison of the LDA and GGA results on Fig. 2 also indicates this trend. Thus, it is likely that strain plays some role in the differences between the LDA and the GGA results.

We now return to our discussion of $V^{(2)}(5)$, for which we observe large discrepancies with different $\mathbf{k}$-point summations (Table 1) and with the LDA vs. the GGA (Fig. 2). Although the exact reason for this large error bar is unclear, one possible explanation is that trio interactions contribute to the total interaction energy of the configuration that we used to obtain $V^{(2)}(5)$. As we see in Fig. 3, this configuration consists of a row of atoms occupying alternating fcc sites along the $\langle 110\rangle$ direction. A trio in 


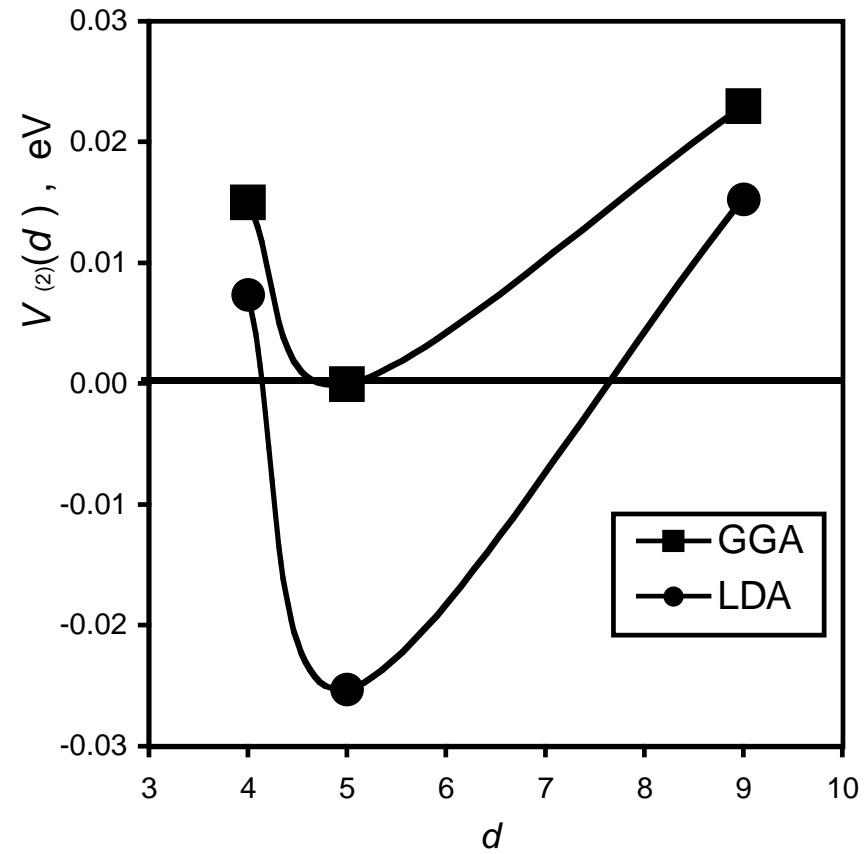

Figure 2. Comparison of LDA and GGA pair-interaction energies $V^{(2)}(d)$ for three different adsorbate separations $d$.

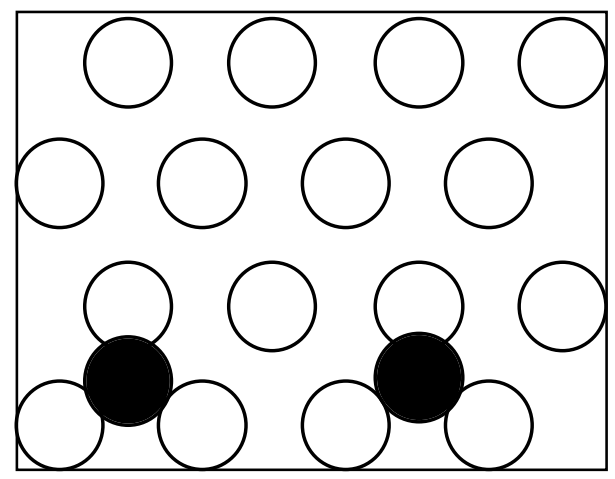

Figure 3. Configuration of adatoms used to obtain $V^{(2)}(5)$.

this configuration would most likely consist of three adatoms in alternating fcc sites along the $\langle 110\rangle$ direction. Although we are not able to quantify this particular trio interaction, we know that the trio interaction $V^{(3)}(4,5,9)$ is attractive by $12 \mathrm{meV}$ [26]. Einstein found in simple, tight-binding calculations that the magnitude of the trio interaction is determined primarily 
by the two, shortest adatom separations in the trio [27]. In light of these results, a non-zero trio interaction seems possible for the adatom configuration in Fig. 3. If this is the case, then one trio interaction is incorporated into our pair parameterization for $V^{(2)}(5)$ and the combined uncertainties in both interactions could lead to the relatively large error bar for this interaction coefficient.

Figure 4 shows the pair interaction for the various distances probed. To facilitate resolution of the long-range interactions, the short-range interactions at distances 1 (the nearest-neighbor fcc site to the central adatom) and 2 ( the second-closest hcp site to the central adatom) are not shown. For both surfaces, the interaction is relatively weak at moderate distances and it is the strongest and the most repulsive at the longest distances probed. For strained $\mathrm{Ag}(111)$ [cf., Fig. 4(a)], the interaction can be as repulsive as $\sim 50 \mathrm{meV}$ at the longest distances and the repulsion forms a ring-like structure around the central adatom. The interactions are weaker on $\operatorname{Ag}(111)$ [Fig. 4(b)] and do not form a complete ring over the distances probed here. Generally, we find that the adatom pair interaction is lower for $\operatorname{Ag}(111)$ than it is for strained $\operatorname{Ag}(111)$ - at all distances for which the pair interaction is appreciable, the attractions on $\mathrm{Ag}(111)$ are stronger and the repulsion is weaker. We note that Bogicevic and co-workers [28] have recently obtained interactions similar to ours in DFT-LDA calculations of $\mathrm{Al}$ on $\mathrm{Al}(111)$ and $\mathrm{Cu}$ on $\mathrm{Cu}(111)$.

Since the surface atoms have the same configuration in all of the results discussed above, the elastic interaction due to adsorbate-induced rearrangement of the surface atoms is suppressed. To assess the role of elastic interactions, we recompute the pair-interaction coefficients in a few trial structures for which both the adsorbate and first-layer surface atoms are fully relaxed until the forces are typically less than $0.025 \mathrm{eV} / \AA$.

In Table 2, we show the elastic contribution to the pair-interaction coefficients at several adsorbate separations. This is given as $\Delta V^{(2)}(d)$, where $\Delta V^{(2)}(d)$ is the difference between the pair-interaction coefficient obtained for a fully relaxed structure and that for the electronic interaction. ¿From Table 2, we see that the elastic interaction plays a very small role in the total interaction energy for $\mathrm{Ag}(111)$. The largest relaxation is for a dimer, a weak attraction of $14 \mathrm{meV}$. The elastic interaction is considerably stronger for strained $\operatorname{Ag}(111)$ and is seen to be appreciable up to distance 5 on Fig. 4. At distances 4 and 5 , the reduction of the pair interaction upon relaxation is greater than the electronic interaction [cf., Fig. 4(a)], indicating that the elastic interaction is dominant at these distances. At longer distances, the elastic interaction is weak and we conclude that the repulsive ring seen on strained $\operatorname{Ag}(111)$ is electronic in origin.

Adsorbate interactions at surfaces can influence both thermodynami- 


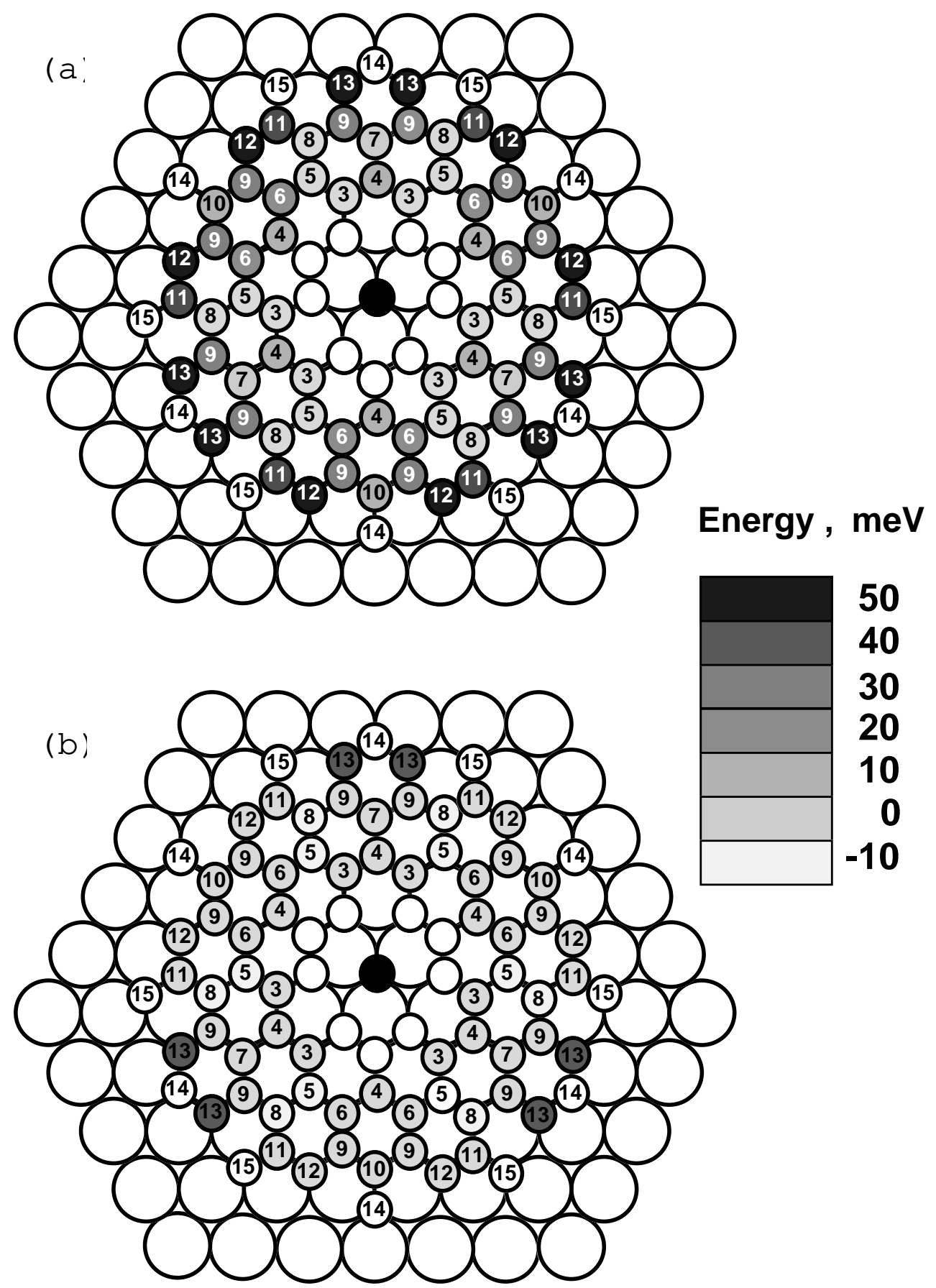

Figure 4. Pair interaction as a function of distance from a central adatom (shown in black) for strained $\operatorname{Ag}(111)$ (a) and unstrained $\operatorname{Ag}(111)$ (b). 
TABLE 2. The elastic contribution $\Delta V^{(2)}(d)$ (units of eV) to the pair interaction between two adsorbates separated by a distance $d$.

\begin{tabular}{ccc}
\hline Distance, $d$ & $\Delta V^{(2)}(d)-\operatorname{Ag}(111)$ & $\Delta V^{(2)}(d)-$ Strained $\operatorname{Ag}(111)$ \\
\hline 1 & -0.014 & -0.051 \\
4 & 0.003 & -0.016 \\
5 & 0.003 & -0.023 \\
9 & 0.003 & -0.007 \\
11 & 0.003 & -0.003 \\
\hline
\end{tabular}

cally favored structures and adsorbate diffusion. To assess the impact of adsorbate interactions on surface diffusion, we obtain the diffusion barrier for an isolated adatom to hop between adjacent three-fold hollow sites on both surfaces. In these calculations, both the adatom and the surface atoms are relaxed. The diffusion barrier is given by the difference between the total energy when the adatom is at a bridge site and when the atom is at a fcc three-fold hollow site. We find for $\operatorname{Ag}(111)$ that the diffusion barrier is 87 meV. For strained $\operatorname{Ag}(111)$, we find a barrier of $52 \mathrm{meV}$. Our barriers are in good agreement with those of Ratsch et al. [15], who found $81 \mathrm{meV}$ for $\mathrm{Ag}(111)$ and $63 \mathrm{meV}$ for strained $\mathrm{Ag}(111)$ in DFT-LDA calculations. Differences between the barriers that we find and those found by Ratsch can be attributed to differences in the exchange-correlation functional (LDA vs. the GGA used here). Additionally, there are differences between the lattice constant predicted for the strained $\operatorname{Ag}(111)$ surface in the two studies. As discussed above, the lattice in our GGA calculations is compressively strained by $4.61 \%$ relative to the bulk, while the strain for the LDA system is $3.21 \%$. Ratsch et al. found that the value of the diffusion barrier on a strained $\operatorname{Ag}(111)$ surface is very sensitive to the degree to which the surface is strained and that smaller diffusion barriers arise on surfaces under greater compression [15]. The difference between our diffusion barrier and that found by Ratsch et al. [15] is adherent to this trend. Our diffusion barriers are also in good agreement with experimental values [22] for $\mathrm{Ag}$ on 1-ML-Ag/Pt(111) (60 meV) and on $\mathrm{Ag}(111)$ (97 meV).

Comparing the diffusion barrier for an adatom on strained $\mathrm{Ag}(111)$ to the magnitude of the pair interactions on this surface, we see that the two are comparable. Thus, one conclusion arising from this work is that substrate-mediated interactions can influence adsorbate diffusion and morphologies formed during growth at surfaces. Our recent kinetic Monte Carlo simulation studies of island nucleation and growth show that this is the case $[23,26]$. Recent experimental STM studies have begun to probe the 
role of adsorbate lateral interactions in determining the structure of adsorbate islands at surfaces $[9,29]$. Theoretical-experimental interplay in this area would be fruitful.

\section{Acknowledgements}

This research is supported by the Alexander von Humboldt Foundation and NSF grant No. DMR-9617122.

\section{References}

1. Bradshaw, A. and Scheffler, M. (1979) J. Vac. Sci. Technol.16, 447.

2. Lau, K. H. and Kohn, W. (1977) Surf. Sci.65, 607.

3. Einstein, T. L. and Schrieffer, J. R. (1973) Phys. Rev. B 7, 3629.

4. Einstein, T. L. (1996) Handbook of Surface Science, W. N. Unertl, Ed. (Elsevier, Amsterdam), Vol. 1, p. 577.

5. Lau, K. H. and Kohn, W. (1978) Surf. Sci. 75, 69.

6. Hyldgaard, P. and Persson, M. (2000) J. Phys.: Condens. Matter 12, L13.

7. Briner, B. G., Hoffman, Ph., Doering, M., Rust, H.-P., Bradshaw, A. M., Petersen, L., Sprunger, P. T., Laegsgaard, E., Besenbacher, F., and Plummer, E. W. (1997) Europhysics News 28, 148.

8. Jeandupeux, O., Burgi, L., Hirstein, A., Brune, H., and Kern, K. (1999) Phys. Rev. $B$ 59, 15926.

9. Repp, J., Moresco, F., Meyer, G. and Rieder, K.-H. (2000) Phys. Rev. Lett. 85, 2981.

10. Bockstedte, M., Kley, A., Neugebauer, J. and Scheffler, M (1997) Comput. Phys. Comm. 107, 187.

11. Kleinmann, L and Bylander, D. M. (1982) Phys. Rev. Lett. 48, 1425.

12. Fuchs, M. and Scheffler, M. (1999) Comput. Phys. Commun. 119, 67.

13. Perdew, J. P., Burke, K., and Ernzerhof, M. (1996) Phys. Rev. Lett. 77, 3865.

14. Yu, B.-D. and Scheffler, M. (1997) Phys. Rev. B 5513916.

15. Ratsch, C., Seitsonen, A. P., and Scheffler, M. (1997) Phys. Rev. B 55, 6750.

16. Perdew, J. P., Chevary, J. A., Vosko, S. H., Jackson, K. A., Pederson, M. R., Singh, D. J., and Fiolhais (1992) Phys. Rev. B 46, 6671.

17. Narasimhan, S. and Scheffler, M. (1997) Z. Phys. Chem. 202, 253.

18. Xie, J., de Gironcoli, S., Baroni, S., and Scheffler, M. (1999) Phys. Rev. B 59, 965.

19. Statiris, P., Lu, H. C., and Gustafsson, T. (1994) Phys. Rev. Lett. 3574.

20. Soares, E. A., de Carvalho, V. E., de Castillo, C. M. C., de Carvalho, A. V., Toomes, R., and Woodruff, D. P. (1999) Surf. Sci. 419, 89.

21. Ratsch, C. and Scheffler, M. (1998) Phys. Rev. B 58, 13163.

22. Brune, H., Bromann, K., Röder, H., Kern, K., Jacobsen, J., Stoltze, P., Jacobsen, K., and Norskov, J. (1995) Phys. Rev. B 52, R14380.

23. Fichthorn, K. A. and Scheffler, M. (2000) Phys. Rev. Lett. 84, 5371.

24. Stampfl, C., Kreuzer, H. J., Payne, S. H., Pfnür, H., and Scheffler, M. (1999) Phys. Rev. Lett. 83, 2993.

25. Ceperly, D. M. and Alder, B. (1980) Phys. Rev. Lett. 45, 567.

26. Fichthorn, K. A. and Scheffler, M. (to be published).

27. Einstein, T. L. (1979) Surf. Sci. 84, L497.

28. Bogicevic, A., Ovesson, S., Hyldgaard, P., Lundqvist, B. I., Brune, H., and Jennison, D. R. (To appear in Phys. Rev. Lett.).

29. Österlund, L., Pederson, M. O., Stensgaard, I., Laegsgaard, E., and Besenbacher, F. (1999) Phys. Rev. Lett. 83, 4812. 